\title{
Surgery for the Venous Disaster - Phlegmasia Cerulea Dolens
}

\author{
Chien-Hui Lee, MD, Yung-Kun Hsieh, MD \\ Division of Cardiovascular Surgery, Department of Surgery, Changhua Christian Hospital, Changhua, Taiwan
}

\section{ABSTRACT}

Phlegmasia cerulea dolens is an acute fulminating form of extensive venous thrombosis. Limb loss, post-thrombotic syndrome and life-threatening conditions can occur without appropriate management.

Treatment methods vary; there presently is no consensus on the best form of treatment. Endovascular procedures have been a good option for treating deep vein thrombosis, yet they may be insufficient for patients suffering from phlegmasia cerulea dolens. Venous thrombectomy with the guidance of venography quickly relieves symptoms, hardly causes complications, yields optimal mid-term results, and can be a justifiable treatment for phlegmasia cerulea dolens.

\section{INTRODUCTION}

Phlegmasia cerulea dolens (PCD) is a rare and devastating emergency caused by extensive venous thrombosis. A massive thrombus occludes both deep and superficial veins, obstructs the capillary outflow and then elevates the hydrostatic pressure, leading to fluids shifting into the interstitial space and causing substantial tissue edema. The plasma loss can be immense and hypovolemic shock develops as a consequence [Perkins 1996].

The extreme venous hypertension with massive tissue edema impedes the arterial inflow, compromises the tissue perfusion, and ultimately results in venous gangrene, limb loss and death. This ischemic entity is distinguished from the more common non-ischemic venous thrombosis, phlegmasia alba dolens, and is characterized by cyanosis associated with limb swelling and pain [Perkins 1996; Yang 2016; Klok 2013; Wakefield 2009]. Without timely and adequate management, the potentially reversible PCD may become irreversible venous gangrene in $40 \%$ to $60 \%$ of cases, which bears a $20 \%$ to $50 \%$ amputation rate; the risk of pulmonary embolism increases with tissue necrosis, and a $20 \%$ to $41 \%$ mortality rate has been reported [Perkins 1996; Yang 2016; Patel 1998].

Received March 11, 2019; received in revised form May 6, 2019; accepted May 31, 2019.

Correspondence: Yung-Kun Hsieh, Division of Cardiovascular Surgery, Department of Surgery, Changhua Christian Hospital, Changhua, Taiwan; (e-mail: surgn68@gmail.com).

\section{METHODS}

From May 2016 to May 2018, three patients were diagnosed with PCD in our hospital. They were two males and one female age 49 years to 64 years. All patients had their left lower limbs involved.

\section{Clinical presentations}

PCD was the first presentation in all cases, including sudden swelling, pain, and cyanotic appearance of lower extremities (Figure 1). In every case, a weak or missing Doppler signal in the dorsalis pedis and/or posterior tibia arteries was noted and one of the patients had pain extending to the lower back. Relevant risks were malignancy (2/3) and common iliac vein stenosis (3/3).

We performed computed tomography (CT) imaging directly instead of compression ultrasonography to better identify the arterial or venous lesion as well as the concomitant intra-abdominal pathology. All patients had iliac-femoral-popliteal type deep vein thrombosis (DVT). It extended into the lower inferior vena cava (IVC) in one patient, and in two cases, the blood flow signal below the knee was absent in the arterial phase while present in the venous phase (Figure 2). No patient displayed symptoms of pulmonary embolism, such as chest pain, hemoptysis, or dyspnea.

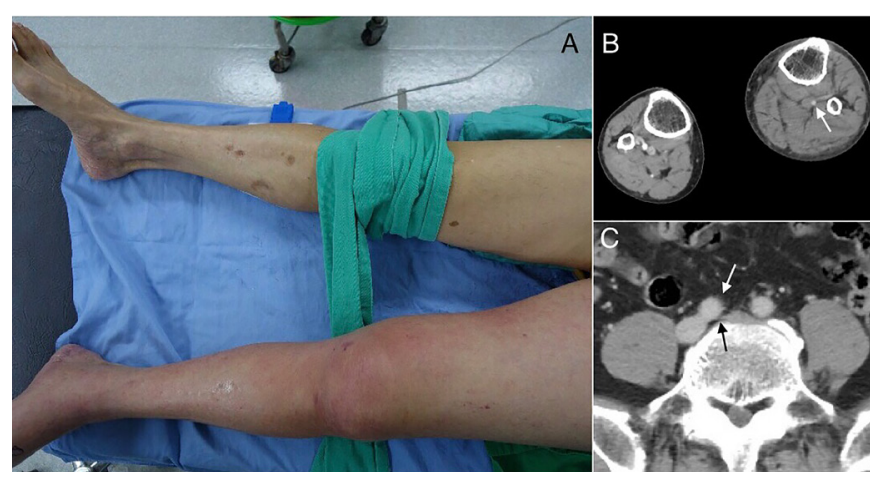

Figure 1. Clinical presentations and characteristic imaging of PCD. The PCD affected limb was cyanotic and had tense swelling (Figure 1A). The $C T$ angiography demonstrated the decreased artery flow in the affected limb (Figure 1B, white arrow) and a stenotic left CIV (Figure $1 \mathrm{C}$, black arrow) compressed by the right $\mathrm{CIA}$ (Figure 1c, white arrow). CIA, common iliac artery; CIV, common iliac vein; $\mathrm{CT}$, computed tomography; PCD, phlegmasia cerulea dolens. 


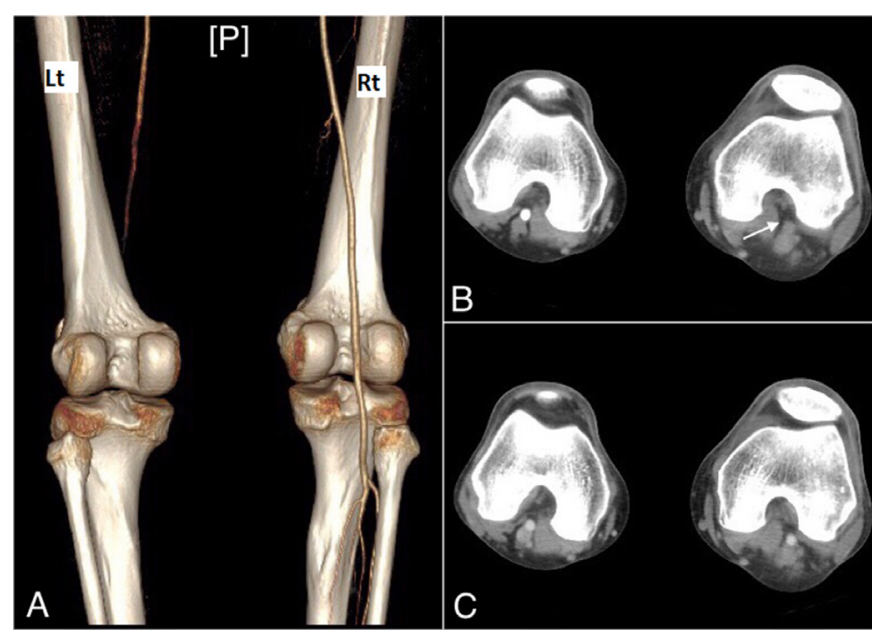

Figure 2. PCD can mimic arterial occlusive disease in CT imaging. In the arterial phase of CT angiography, the slow blood flow caused the signal to disappear in the below-knee arteries of the PCD affected limb (Figure 2A, white arrow in Figure 2B), but the signal was detected in the venous phase (Figure $2 \mathrm{C}$ ).

CT, computed tomography; Lt, left; Rt, right; P, posterior; PCD, phlegmasia cerulea dolens.

\section{Treatment methods}

Fluid resuscitation, anticoagulation, and affected limb elevations were provided as the initial treatment for all patients. The regiment of anticoagulation in our institution is intravenous heparin at an initial bolus of $80 \mathrm{U} / \mathrm{kg}$ followed by a continuous intravenous infusion, with dose adjustment to target a partial thromboplastin time prolongation (50 sec $70 \mathrm{sec}$ ).

Subsequent management included open venous thrombectomy with the aid of DSA. All patients were well informed about the disease pattern and consented to our surgical treatment policy.

\section{Venous thrombectomy}

Venous thrombectomy was performed through the left common femoral vein (CFV) under general anesthesia with the aid of a fluoroscope. After exploring and controlling the $\mathrm{CFV}$, we transversely incised the vein and delivered a 0.035 guide wire up to the IVC under DSA. Proximal thrombus was removed by a dual lumen Fogarty catheterization, and distal thrombectomy was performed by the same method until the competent venous valve impeded the advance of the Fogarty catheter (Figure 3). The more distal segment of thrombus was pushed out by squeezing the lower extremity externally. The Fogarty catheter was replaced by an $8 \mathrm{~F}$ catheter, through which we performed the intra-operative venography by manually injecting the contrast medium. During the same operation, the abovementioned procedures (thrombectomiesvenography) may have been repeated with the presence of residual thrombus.

For associated venous lesions, transluminal angioplasty was performed through the catheter to assure the venous inflow and to dilate the concomitant stenosis in the iliac vein. IVC filter deployment or catheter-directed thrombolysis (CDT) was required when unignorable stenosis or residual thrombus persisted. The incision of the common femoral vein was sutured closed after the procedure.

All patients were under close surveillance in the intensive care unit and received fluid resuscitation, electrolytes, and renal function monitoring. Heparin infusion was continued and overlapped with oral anticoagulation (warfarin or Rivaroxaban). Oral anticoagulant treatment was kept for at least three months. It was discontinued six months later in one patient because he was symptom-free and had no residual venous lesions on the follow-up imaging study. One patient continued receiving Rivaroxaban as the risk of malignancy persisted.

The outcomes of affected limbs were assessed by clinical pictures and imaging studies (compressive ultrasonography or CT angiography).

\section{RESULTS}

All patients had stenotic lesions ( $>50 \%)$ in the left common iliac vein and underwent concomitant endovascular angioplasty. Limb congestion and pain were alleviated immediately after the surgeries. Foot arterial pulses returned to normal, and no perioperative pulmonary emboli occurred.

One patient died of terminal malignancy two months later. An IVC filter was deployed in one patient because the thrombus extended into the distal IVC and intra-operative venography demonstrated residual thrombus persisted after thrombectomies (Figure 4). A multiple side-hole catheter was left in the common iliac vein for Urokinase infusion and was retrieved two days later.

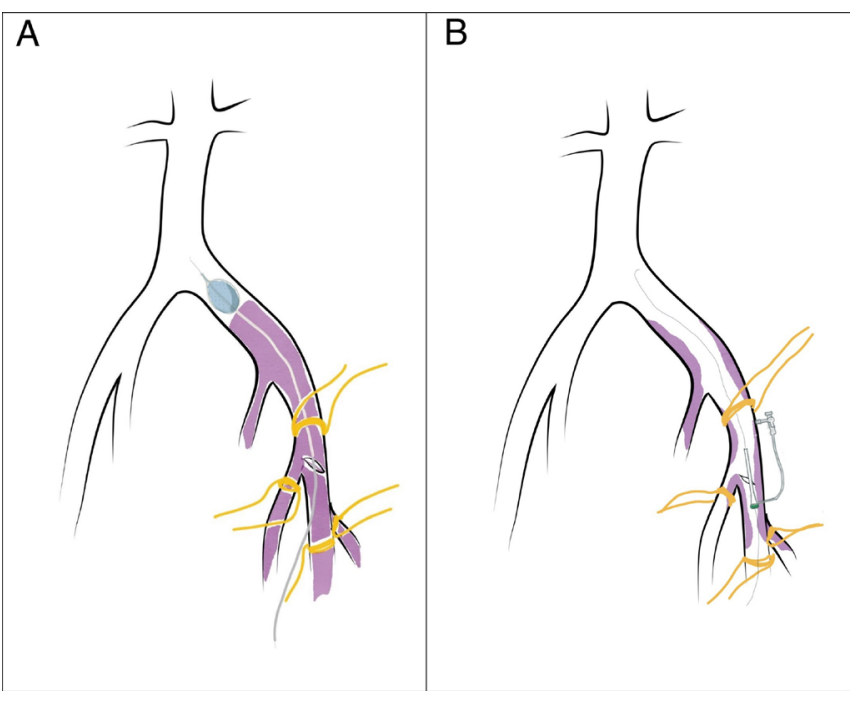

Figure 3. Surgical procedures. We transversely incised the CFV and delivered a guide wire up to the IVC under DSA. The proximal thrombus was removed by a dual lumen Fogarty catheter, while distal thrombus was pushed out by squeezing the lower extremity. The Fogarty catheter was replaced by an $8 \mathrm{~F}$ catheter, through which we injected contrast medium manually to perform a venography. CFV, common femoral vein; IVC, inferior vena cava; DSA, digital subtraction angiography 


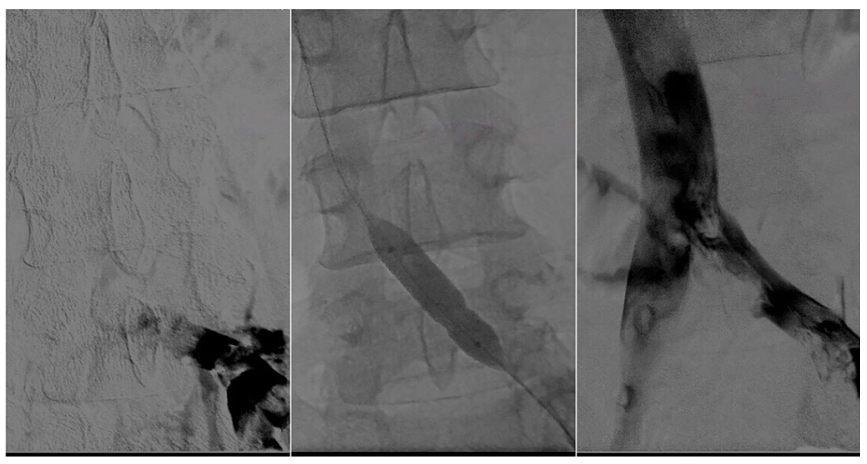

Figure 4. Concomitant percutaneous transluminal angioplasty was performed with the presence of venous stenosis.

From left to right on the panel: The thrombus extended from the lower IVC to the left popliteal vein, and the patient underwent venous thrombectomy with the aid of DSA (the left figure). Concomitant percutaneous transluminal angioplasty was performed to dilate the stenotic iliac vein (the middle figure). Residual thrombus still presented after repeated thrombectomies (the right figure). DSA, digital subtraction angiography; IVC, inferior vena cava.

The patients were followed up for two months to two years (mean duration: 9.6 months). Besides one mortality due to malignancy, the other two had no swelling of their affected extremities, no recurrence of DVT and required no secondary intervention. Details of the patients, interventions, and outcomes are summarized in Table 1.

\section{DISCUSSION}

The majority of PCDs occur in the fifth and sixth decades of life [Perkins 1996]. The left lower limbs are involved more frequently than the right lower limbs (45\% vs 29\%), and both lower limbs are affected in $26 \%$ of cases [Perkins 1996; Chinsakchai 2011].

The most well-known risk factors are malignancy, which presents in $1 / 3$ of the cases, and an underlying hypercoagulable state [Perkins 1996]. Other precipitating factors are postoperative or post-traumatic status, postpartum status, use of contraceptive agents, heparin-induced thrombocytopenia, history of DVT, May-Thurner syndrome, prolonged immobility and a variety of inflammatory conditions; approximately 10\% 16\% of patients have no known risk factors [Perkins 1996; Klok 2013; Chinsakchai 2011; Zhang 2018; Ikegami 2016; Onuoha 2015; Mumoli 2012; Warkentin 1997].

In our study all patients had their left lower extremities involved, two had malignancy, and one had May-Thurner syndrome.

The diagnosis is usually clinical. Doppler ultrasound is the recommended tool to assess the pulse of the edematous limbs and exclude the possibility of arterial occlusion [Perkins 1996; Yang 2016; Klok 2013; Chinsakchai 2011]. Venography may be problematic in assessing PCD when the contrast agent is impeded in totally occluded situations [Perkins 1996; Kalagher 2015]. Magnetic resonance imaging and CT are helpful in investigating both vascular and associated intra-abdominal pathology. However, slow arterial blood flow can mimic occlusion and confound the interpretation [Kalagher 2015], which happened in two of our cases (Figure 2). The venous phase of CT is then required for asserting the diagnosis.

There is no definite treatment, but the goal of management is invariable: prevent thrombus propagation, release the venous hypertension, and preserve the collateral circulation and tissue viability [Perkins 1996; Chinsakchai 2011]. The therapeutic strategy depends largely on the clinical picture and experience of the surgeon, and it should be more aggressive with greater extent of tissue ischemia and venous gangrene [Chinsakchai 2011; Rutherford 1997].

Table 1 Summary of the patients' profiles, interventions and outcomes.

\begin{tabular}{|c|c|c|c|c|c|}
\hline \multicolumn{6}{|c|}{ Images } \\
\hline Case & Comorbidities & Presentations & Thrombus Lesions & Definite treatment & Outcomes \\
\hline (M) $60 y$ & $\begin{array}{c}\text { May-Thurner syndrome } \\
\text { Diabetes }\end{array}$ & $\begin{array}{l}\text { Lt lower limb: cyanosis, } \\
\text { tense, cold, swelling }\end{array}$ & $\begin{array}{c}\text { Lt CIV to PopV } \\
\text { Lt CIV and EIV stenosis }\end{array}$ & $\begin{array}{c}\text { VT } \\
\text { PTA on Lt EIV CIV and EIV } \\
(8 \mathrm{~mm} \text { balloon })\end{array}$ & $\begin{array}{c}\text { Fair* } \\
\text { 2nd intervention: no }\end{array}$ \\
\hline (F) $64 y$ & $\begin{array}{l}\text { Malignancy } \\
\text { Renal failure Diabetes }\end{array}$ & $\begin{array}{c}\text { Lt lower limb: cyanosis, } \\
\text { tense, and swelling }\end{array}$ & $\begin{array}{c}\text { Lt CIV to PopV } \\
\text { Lt CIV and EIV stenosis }\end{array}$ & $\begin{array}{l}\text { VT } \\
\text { PTA on Lt CIV (10mm balloon), } \\
\text { Lt EIV (7mm balloon); IVC filter } \\
\text { deployment }\end{array}$ & $\begin{array}{l}\text { Expired two months later of } \\
\text { terminal stage malignancy. } \\
\text { 2nd intervention: no }\end{array}$ \\
\hline (M) $49 y$ & Malignancy & $\begin{array}{c}\text { Lt lower back pain } \\
\text { Lt lower limb: cyanosis, } \\
\text { cold, swelling }\end{array}$ & $\begin{array}{c}\text { Lower IVC to Lt PopV } \\
\text { Lt CIV stenosis }\end{array}$ & $\begin{array}{c}\text { VT } \\
\text { PTA on Lt CIV (12mm balloon); } \\
\text { CDT in Lt CIV }\end{array}$ & $\begin{array}{c}\text { Fair* } \\
\text { 2nd intervention: no }\end{array}$ \\
\hline
\end{tabular}

CIV, common iliac vein; EIV, external iliac vein, EVRF, endovenous radiofrequency ablation, F, female; GSV, great saphenous vein; Lt, left; M, male; PopV, popliteal vein; PTA, percutaneous transluminal angioplasty, VT, venous thrombectomy; y, years old.

*Fair means "No limb edema and no recurrent venous thrombosis" 
The first-aid management consists of fluid resuscitation, anticoagulation, and adequate limb elevation [Perkins 1996; Chinsakchai 2011; Onuoha 2015]. For those who fail to respond with systemic anticoagulation within $6 \mathrm{hrs} \sim 12 \mathrm{hrs}$, vascular specific interventions may be beneficial [Perkins 1996; Chinsakchai 2011; Weaver 1988]. On occasion, the presence of compartment syndrome associated with progressive gangrene necessitates emergent fasciotomy, and the amputation rate is highest in this circumstance [Perkins 1996; Chinsakchai 2011; Onuoha 2015].

Surgical thrombectomy correlates well with the treatment goal by promptly reducing the thrombus load and decreasing the compartment pressure. This prevents pulmonary embolism and venous gangrene and also achieves good mid-term and long-term outcomes [Perkins 1996; Yang 2016; Chinsakchai 2011; Zhang 2018; Comerota 2012; Casey 2012; Goyal 2013]. However, poor clearance of distal thrombus, high re-thrombotic rates, and the possibility of minimal improvement in post-phlebitis syndrome due to a venous valve injury make an open thrombectomy less preferable [Perkins 1996; Chinsakchai 2011; Zhang 2018; Laohapensang 2013; Lansing 1968]. CDT with or without percutaneous mechanical thrombectomy is less invasive and has been the preferred management for DVT [Perkins 1996; Zhang 2018; Amin 2014; Meissner 2012; Jaff 2011]. Nevertheless, endovascular interventions for PCD can be limited by ineffectiveness in clearing totally occluded venous segments and an increase in the incidence of pulmonary embolism resulting from manipulating the catheter and clot fragmentation during the procedure [Perkins 1996; Chinsakchai 2011; Thery 1990]. The time-consuming nature can defer tissue reperfusion and thereby worsen the outcome.

Combining open thrombectomy with endovascular techniques not only possesses merits of open thrombectomy, but also avoids later venous insufficiency. The hybrid strategy proves valuable and may be a potential option when treating ischemic limbs affected by PCD [Yang 2016; Zhang 2018; Laohapensang 2013; Igari 2014; Sarwar 2009; Norgren 2007; Mewissen 1999].

We suggest an aggressive attitude of venous thrombectomy for PCD with the aid of DSA, combined with angioplasty or CDT for associated lesions. From our experiences, this hybrid technique quickly dredged the inflow channel and dilated the stenotic venous segments so that the congestion was resolved and disease recurrence was prevented.

In our cases, swelling immediately was resolved and no patient so far has displayed post-thrombotic syndrome.

\section{CONCLUSION}

Rapid restoration of tissue perfusion is the principle management for PCD. Interventions usually are required to keep the patency of venous outflow and to prevent gangrene.

In the current study, three PCD patients were treated successfully with open thrombectomy under DSA combined with angioplasty or CDT for associated lesions. This hybrid strategy is considered as a treatment option for the management of PCD. However, further research on its effectiveness in a larger population of patients is needed.
REFERENCES

Amin VB, Lookstein RA. 2014. Catheter-directed interventions for acute iliocaval deep vein thrombosis. Tech Vasc Interv Radiol 17(2):96-102.

Casey ET, Murad MH, Zumaeta GM, et al. 2012. Treatment of acute iliofemoral deep vein thrombosis. J Vasc Surg 55(5):1463-1473.

Chinsakchai K, Ten Duis K, Moll FL, de Borst GJ. 2011. Trends in management of phlegmasia cerulea dolens. Vasc Endovascular Surg 45(1):5-14.8.

Comerota AJ. 2012. The current role of operative venous thrombectomy in deep vein thrombosis. Semin Vasc Surg 25(1):2-12.

Goyal VD, Gupta B, Choudhary U, Maheshwari P, Iyer D. 2013. Venous thrombectomy in cases of acute deep vein thrombosis presenting as acute limb ischemia (Phlegmasia Cerulea Dolens): report of 2 cases and review of literature. Indian J Thorac Cardiovasc Surg 30(1):36-9.

Igari K, Kudo T, Toyofuku T, Jibiki M, Inoue Y. 2014. Surgical thrombectomy and simultaneous stenting for deep venous thrombosis caused by iliac vein compression syndrome (May-Thurner syndrome). Ann Thorac Cardiovasc Surg 20(6):995-1000.

Ikegami T, Kuriyama A, Matsukubo S. 2016. Phlegmasia cerulea dolens. QJM. doi: 10.1093/qjmed/hcw016.

Jaff MR, McMurtry MS, Archer SL, et al. 2011. Management of massive and submassive pulmonary embolism, iliofemoral deep vein thrombosis, and chronic thromboembolic pulmonary hypertension: a scientific statement from the American Heart Association. Circulation 123(16):1788-1830.

Kalagher SD, Kane DD. 2015. Phlegmasia cerulea dolens: before and after lysis. Intern Emerg Med 10(1):103-104.

Klok FA, Huisman MV. 2013. Seeking optimal treatment for phlegmasia cerulea dolens. Thromb Res 131(4):372-3.

Lansing AM, Davis WM. 1968. Five-year follow-up study of iliofemoral venous thrombectomy. Ann Surg 168(4):620-628.

Laohapensang K, Hanpipat S, Aworn S, Orrapin S. 2013. Surgical venous thrombectomy for phlegmasia cerulea dolens and venous gangrene of the lower extremities. J Med Assoc Thai 96(11):1463-1469. PMID: 24428096.

Meissner MH. 2012. Rationale and indications for aggressive early thrombus removal. Phlebology 27 Suppl 1:78-84.

Mewissen MW, Seabrook GR, Meissner MH, Cynamon J, Labropoulos N, Haughton SH. 1999. Catheter-directed Thrombolysis for Lower Extremity Deep Venous Thrombosis: Report of a National Multicenter Registry. Radiology 211(1):39-49.

Mumoli N, Invernizzi C, Luschi R, Carmignani G, Camaiti A, Cei M. 2012. Phlegmasia cerulea dolens. Circulation 125(8):1056-1057.

Norgren L, Hiatt WR, Dormandy JA, Nehler MR, Harris KA, Fowkes FG; TASC II Working Group. 2007. Inter-Society Consensus for the Management of Peripheral Arterial Disease (TASC II). J Vasc Surg doi: 10.1016/j. jvs.2006.12.037.

Onuoha CU. 2015. Phlegmasia Cerulea Dolens: A Rare Clinical Presentation. Am J Med 128(9):e27-28.

Patel NH, Plorde JJ, Meissner M. 1998. Catheter-directed thrombolysis in the treatment of phlegmasia cerulea dolens. Ann Vasc Surg 12(5):471-475.

Perkins JM, Magee TR, Galland RB. 1996. Phlegmasia caerulea dolens and venous gangrene. Br J Surg 83(1):19-23.

Rutherford RB, Baker JD, Ernst C, et al. 1997. Recommended standards for reports dealing with lower extremity ischemia: revised version. J Vasc Surg 26(3):517-538. 
Sarwar S, Narra S, Munir A. 2009. Phlegmasia cerulea dolens. Tex Heart Inst J 36(1):76-77.

Thery C, Asseman P, Amrouni N, et al. 1990. Use of a new removable vena cava filter in order to prevent pulmonary embolism in patients submitted to thrombolysis. Eur Heart J 11(4):334-341.

Wakefield TW. 2009. Treatment algorithm for acute deep venous thrombosis:

current guidelines. In: Gloviczki P (eds) Handbook of venous disorders. Hodder Arnold, London. pp 265-76.
Warkentin TE, Elavathil LJ, Hayward CP, Johnston MA, Russett JI, Kelton JG. 1997. The pathogenesis of venous limb gangrene associated with heparin-induced thrombocytopenia. Ann Intern Med 127(9):804-812.

Weaver FA, Meacham PW, Adkins RB, Dean RH. 1988. Phlegmasia cerulea dolens: therapeutic considerations. South Med J 81(3):306-312.

Yang SS, Yun WS. Surgical Thrombectomy for Phlegmasia Cerulea Dolens.2016.Vasc Specialist Int 32(4):201-4.

Zhang X, Chen Z, Sun Y, Xu M. 2018. Surgical Thrombectomy and Simultaneous Stenting for Phlegmasia Cerulea Dolens Caused by Iliac Vein Occlusion. Ann Vasc Surg 51:239-245. 